seronegative late-onset RA. Articular erosions developed in $35 \%$ of the seronegative lateonset RA group compared with none of the PMR group.

According to the authors' diagnostic algorithm, patients who present with a plasma viscosity of at least 1.91 (normal value $<1.73$ ) and without combined wrist, proximal interphalangeal and/or metacarpophalangeal joint involvement have an $88 \%$ probability of a diagnosis of PMR (odds ratio 7.396). These findings are consistent with RF-negative RA and PMR being distinct clinical entities.

Original article Pease CT et al. (2008) Polymyalgia rheumatica can be distinguished from late onset rheumatoid arthritis at baseline: results of a 5 -yr prospective study. Rheumatology (Oxford) [doi:10.1093/rheumatology/ken343]

\section{Influence of infliximab on cardiovascular risk factors in patients with RA}

The prevalence of cardiovascular disease (CVD) is increased in patients with rheumatoid arthritis (RA), even after adjustment for traditional CVD risk factors. Wong et al. have now performed a post-hoc analysis that suggests long-term infliximab therapy significantly decreases arterial stiffness - one of multiple CVD risk factors - in patients with RA, which seems to be independent of traditional CVD risk factors, and might help to explain the beneficial effects of tumor necrosis factor inhibitors on cardiovascular risk in these patients.

Wong et al. randomly allocated 17 patients to receive $3 \mathrm{mg} / \mathrm{kg}$ of intravenous infliximab (at weeks 0,2 , and 6 , and then every 8 weeks up to week 54) and 9 patients to receive placebo. After 16 weeks, all placebo-treated patients opted to receive infliximab. Measures of cardiovascular risk and RA disease activity were recorded for up to 56 weeks. Post-hoc analyses suggested that infliximab-treated patients had a significant reduction in arterial stiffness, as measured by pulse wave velocity, but no change in the other CVD risk factors assessed. Blood pressure remained stable throughout the study, which is important as it influences pulse wave velocity.

The findings are limited by the small sample size and the fact that all patients in the placebo arm switched to infliximab after 16 weeks. Lipid profiles, adiponectin levels and homeostasis model assessment scores were not recorded until 24 weeks into the study, which meant that the early effects of infliximab on vascular structure could not be examined. All participants received concomitant methotrexate ( $\leq 25 \mathrm{mg} /$ week), but the authors attributed cardiovascular changes to infliximab.

Original article Wong $\mathrm{M}$ et al. (2008) Infliximab improves vascular stiffness in patients with rheumatoid arthritis. Ann Rheum Dis [doi:10.1136/ard.2007.086157]

\section{Lovastatin absorbed through the skin accelerates fracture healing in rats}

Statins increase the expression and secretion of bone morphogenetic protein 2 (BMP2), which stimulates bone formation. As oral statins undergo first-pass hepatic metabolism, however, standard pharmacological doses given to reduce cholesterol do not attain the serum concentration necessary to increase BMP2 concentration. Gutierrez et al. have now demonstrated that lovastatin delivered transdermally - at doses markedly lower than would be required by the oral route-enhances fracture healing in rats.

Weight-matched, 2-month-old rats received a uniform, pinned, femoral fracture, and were treated with transdermal lovastatin applied to the dorsal region $(0.1-5 \mathrm{mg} / \mathrm{kg}$ per day), lovastatin by oral gavage (5-25 mg/kg per day) or placebo for 5 days. Radiological evaluation demonstrated almost $40 \%$ faster fracture repair in rats treated with transdermal lovastatin compared with vehicle-treated rats. Furthermore, at week 6, treatment with transdermal lovastatin, but not oral lovastatin, increased bone mineral density compared with control.

Similarly to other studies of statins and growth factors in fracture healing, the classic sigmoidal dose-response curve was not produced by lovastatin in this study. The reason for this is not entirely clear; it could be related to the counter-regulatory mechanisms in growth cascades when high concentrations of drug are used, or that drug receptors become saturated at low concentrations.

Original article Gutierrez GE et al. (2008) Transdermal lovastatin enhances fracture repair in rats. J Bone Miner Res 23: $1722-1730$ 